\title{
Bounding Greybody and weak deflection angle of NED black holes with many horizons
}

\author{
Wajiha Javed, ${ }^{1, *}$ Iqra Hussain, ${ }^{1, \dagger}$ and Ali Övgün ${ }^{2, \ddagger}$ \\ ${ }^{I}$ Division of Science and Technology, University of Education, Township-Lahore, Pakistan \\ ${ }^{2}$ Physics Department, Eastern Mediterranean University, \\ Famagusta, 99628 North Cyprus via Mersin 10, Turkey.
}

(Dated: November 18, 2021)

\begin{abstract}
In this project, our foremost intention is to analyze the angle of deflection of photon by NED black holes with many horizons in the context of non-linear electrodynamics. For the accomplishment of our intention at first we manipulated the optical metric of the desired black holes for the calculation of Gaussian curvature. After that, the angle of deflection in leading orders is attained from the desired black holes in weak field limit by using the famous Gauss-Bonnet theorem. Moreover, we evince the effect of plasma medium on the angle of deflection. At last, the graphical impact of the angle of deflection for NED black holes with many horizons is examined with respect to black hole charge and impact parameter in both the plasma and non plasma medium. In addition, we discuss the rigorous bound for grey body factor of the desired black hole and also observer its graphical behavior.
\end{abstract}

PACS numbers: 95.30.Sf, 98.62.Sb, 97.60.Lf

Keywords: Relativity; Gravitation lensing; Black hole; Nonlinear electrodynamics; Gauss-Bonnet theorem; Deflection angle; Plasma medium

\section{INTRODUCTION}

As stated by the theory of general relativity (GR), the fundamental of the gravitational lensing (GL) is the deflection of photon by the gravitational field (GF) in the weak field approximation [1,2]. Initial test of GR as soon as 1921, was the gravitational deflection of photon due to the Sun $[3,4]$. GL is a user-oriented method and in this process gravity deflects light. It is very accommodating and obliging for the acknowledgment of the dark matter, the mystifying indiscernible substance that evidently constitute five-sixths of all matter in the universe. As early as 2015, the discovery of gravitational waves by LIGO proclaimed that the theoretical predictions and the observational phenomena were well suited with each other [5]. GL has procured a great attention in different space times and it has been performed by the exertion of different techniques [6]-[10]. GL has turned out to be a well-build method in examining numerous astrophysical objects. In the past, GL was utilize to probe super massive black hole co evolution. In the beginning , the black holes (BHs) were known as "collapsar" (short for collapsed star) the term originated from stellar collapse, later on John Wheeler in 1967 introduced the term BH. An important conjecture given by GR in 1915 was the light must be reflected by the gravitational lens placed in between light source $\mathcal{S}$ and an observer $\mathcal{O}$ as the photon proceeds to an observer and this process was given the name of GL, after sometime the theory was verified with the help of experiments [11,12]. A BH is a region of spacetime having such a strong gravity that it is impossible for the electromagnetic radiations like light and even for the the particles to escape from it. Across the event horizon the gravity remains constant. According to GR prediction an adequate compact mass can deform spacetime for the formation of BH. Owning to the property that $\mathrm{BH}$ reflects no light it behaves as an ideal black body .

Non linear electrodynamics (NLE) theory was initially presented by Max Born [14]. Later on the theory was extended with the cooperation of Leopard Infield [15] to the theory known as Born-Infield theory (BIT). After half century BIT was reestablished as string theory [16]. Space time singularities causes numerous problems in GR. As a consequence, BH contain singularities and horizon. In order to overcome these problems from the BH different techniques are applied such as gravity modification, NLE etc. By using NLE there is a gate way to get rid of these singularities computing the regular BH solution [17]-[20].

Main aim in this paper is to work out the deflection angle of BH with many horizons [21]. To find the angle of deflection Gauss Bonnet Theorem (GBT) will be applied on the desired BH. Currently, Gibbon and Werner (GW) come upon with the new process known as GBT to calculate the angle of deflection of photon [22]. Moreover, it was also proclaimed by them that this process provides an exact result for Schwarzschild BH spacetime in the weak limit approximation (WLA). Afterwards, it has been demonstrated that GBT has produce fruitful results for computing the angle of deflection of various BHs [23]-[71].

\footnotetext{
*Electronic address: wajiha.javed@ue.edu.pk

${ }^{\dagger}$ Electronic address: iqrahussain057@gmail.com

${ }^{\ddagger}$ Electronic address: ali.ovgun@emu.edu.tr
} 
Additionally, let us discuss the GBT in few words. In this theorem, the topological surfaces are connected. In the initial step, Euler characteristic and Riemannian metric is utilized and the surface domain can be selected in order to obtain the Gaussian curvature. The expression for GBT is given as [22]

$$
\iint_{Z_{R}} \mathcal{K} d S+\oint_{\partial Z_{R}} \kappa d t+\Sigma_{i} \theta_{i}=2 \mathcal{X}\left(Z_{R}\right) .
$$

Here $\mathcal{K}$ represents the optical Gaussian curvature. Optical surface is represented by dS. Here the the photon ray bounded the domain except the lens. Here it is expected that the light source $\mathcal{S}$ and the observer $\mathcal{O}$ both are at a coordinate distance from the focal point. Gaussian optical curvature that has been calculated is used to determine the angle of deflection in the weak field limit approximation that can be expressed as follow [22]

$$
\gamma=-\int_{0}^{\pi} \int_{\frac{b}{r \sin \phi}}^{\infty} \mathcal{K} d S
$$

$\gamma$ is representing the angle of deflection. To obtain the $\gamma$ we integrate the Gaussian optical curvature for the infinite surface $\left(B_{R}\right)$. Authors examined the influence of plasma medium on GL [72] and the influence of plasma medium on the shadows of wormholes WHS and BHs was also deliberated by him [73]-[76]. Moreover, Turimov et al. studied the conduct of GL in plasma medium [77]. Furthermore, the influence of the plasma medium on GL and also the shadows of BH were probed by Chakrabatry et al. and by Atamurotov et al. [78, 79]. In addition, Hensh et al. computed the GL of Kehagias-Stefsos dense particles under the influence of plasma [80].

In 1974, Stephen Hawking showed that a BH can emitted quantum radiations, and these radiations are known as hawking radiations (HR)[81]. This fact was derived after studying the quantum field theory (QFT) in a BH. From QFT there is a possibility of creation and inhalations of particle. If the pair production occurs near the BH horizon, one of particle from pair production falling in, with the other moving away from the BH, an observer from outside see this particle as HR [82]. A BH curves a spacetime around it and according to GR, this space time act as a gravitational potential under which particle move. Some of them are reflected back into $\mathrm{BH}$ and other are transmitted by $\mathrm{BH}$ [83]-[88]. Therefore, the HR observed by the observer from outside of BH is different from the spreading by gravitational potential. This difference is called gray body factor (GBF) [89]. There have been a number of studies devoted to calculate GBF such as WKB approximation method [89]-[93]. Another fascinating methods are to bound the GBF from below [94]-[102]

The project is structured in the following manner. We started by the brief review of the basics of the desired spacetime on which we will work. After that, optical geometry and the optical curvature will be calculated. Next section will be devoted for the calculation of angle of deflection of the desired black hole in weak field limit by using GBT. After that, we plot the graphs and examine the graphical behavior of the calculated angle of deflection in detail without plasma medium. The next section contains the calculations of the angle of deflection of photon for plasma medium. Sec. 5 is basically for the determination of the graphical impact for plasma medium on the desired BH. Moreover we worked out the equation for the lower bound of GBF of the $\mathrm{BH}$ and also study its graphical conduct. The last section is the conversation regarding to the conclusions got from the current work and the outcomes obtained from the graphical analysis.

\section{NED BLACK HOLES WITH MANY HORIZONS}

We begin this section with the brief review of the BHs in gravity. An action of gravity coupled with NLE can be represented as [104]

$$
I=\frac{1}{16 \pi} \int d^{4} x \sqrt{-g}(R+H(\varphi)) .
$$

where

$$
\varphi=F_{\mu \nu} F^{\mu \nu}, F_{\mu \nu}=\nabla_{\mu} A_{\nu}-\nabla_{\nu} A_{\mu}
$$

Here, Ricci scalar is described by $R, A_{\mu}$, describes the Maxwell field, and $g$ is for gravity. $H(\varphi)$ is defined as function of $\varphi$. The NED black holes with static and spherically spacetime has the following form [21],

$$
d s^{2}=-Z(r) d t^{2}+\frac{d r^{2}}{Z(r)}+r^{2} d \Omega_{2}^{2},
$$


$Z(r)$ is defined as follow,

$$
Z(r)=1-\frac{2 M}{r}+\frac{Q^{2}}{r^{2}}+\frac{2 a_{2} Q^{4}}{5 r^{6}}, \quad d \Omega_{2}^{2}=d \theta^{2}+\sin ^{2} \theta d \phi^{2}
$$

here $M$ represents the $\mathrm{BH}$ mass, $\mathrm{BH}$ charge is represented by $Q$ and $a_{2}$ indicates the coupling constant. Now inserting the value of $Z(r)$ in Eq.(2) we obtained following expression

$$
\begin{aligned}
d s^{2} & =-\left(1-\frac{2 M}{r}+\frac{Q^{2}}{r^{2}}+\frac{2 a_{2} Q^{4}}{5 r^{6}}\right) d t^{2}+\left(1-\frac{2 M}{r}+\frac{Q^{2}}{r^{2}}\right. \\
& \left.+\frac{2 a_{2} Q^{4}}{5 r^{6}}\right)^{-1} d r^{2}+r^{2} d \theta^{2}+r^{2} \sin ^{2} \theta d \phi^{2} .
\end{aligned}
$$

For static and spherically spacetime we bound ourselves to the equatorial plane $\left(\theta=\frac{\pi}{2}\right)$. We should impose the condition $d s^{2}=0$ to acquire $\mathrm{BH}$ optical metric

$$
d t^{2}=\frac{d r^{2}}{\left(1-\frac{2 M}{r}+\frac{Q^{2}}{r^{2}}+\frac{2 a_{2} Q^{4}}{5 r^{6}}\right)^{2}}+\frac{r^{2} d \phi^{2}}{1-\frac{2 M}{r}+\frac{Q^{2}}{r^{2}}+\frac{2 a_{2} Q^{4}}{5 r^{6}}}
$$

The Gaussian curvature can be calculated by using the following expression

$$
\mathcal{K}=\frac{R_{r \phi r \phi}}{2}
$$

At the end after simplifying we compute the Gaussian optical curvature of the optical metric for the given $\mathrm{BH}$ that is expressed as

$$
\begin{aligned}
\mathcal{K} & =\frac{-2 M}{r^{3}}+\frac{3 Q^{2}}{r^{4}}+\frac{42 a_{2} Q^{4}}{5 r^{8}}+\frac{3 M^{2}}{r^{4}}-\frac{6 M Q^{2}}{r^{5}} \\
& -\frac{76 M a_{2} Q^{4}}{5 r^{9}}+\frac{2 Q^{4}}{r^{6}}+\frac{36 Q^{8} a_{2}}{5 r^{10}}
\end{aligned}
$$

and so, it can be rewritten in leading order terms as

$$
\mathcal{K}=\frac{-2 M}{r^{3}}+\frac{3 Q^{2}}{r^{4}}+\frac{42 a_{2} Q^{4}}{5 r^{8}}+\frac{3 M^{2}}{r^{4}}-\frac{6 M Q^{2}}{r^{5}}+\frac{2 Q^{4}}{r^{6}}
$$

\section{CALCULATION OF WEAK DEFLECTION ANGLE OF NED BLACK HOLES WITH MANY HORIZONS BY GAUSS-BONNET THEOREM}

According to GBT theory the topology of the region $\left(\mathcal{B}_{R}\right)$ and the intrinsic geometry of the spacetime are linked having boundary $\partial \mathcal{B}_{R}$. Now, by the use of GBT we can compute the angle of deflection of the NED black holes with many horizons as follows [22]:

$$
\iint_{\mathcal{B}_{R}} \mathcal{K} d S+\oint_{\partial \mathcal{B}_{R}} k d t+\sum_{j} \epsilon_{j}=2 \pi \mathcal{X}\left(\mathcal{B}_{R}\right)
$$

Optical Gaussian Curvature is represented by $\mathcal{K}$ and $k$ is termed as geodesic curvature declared as $k=\bar{d}\left(\nabla_{\dot{\gamma}} \dot{\gamma}, \ddot{\gamma}\right)$ in a manner that $\bar{d}(\dot{\gamma}, \dot{\gamma})=1$. If $\gamma$ supposed to be a smooth curve then $\dot{\gamma}$ is a unit speed vector. The corresponding exterior angle is $\epsilon_{j}$ at the jth vertex. When it is assumed that $R \rightarrow \infty$, then the respective jump angles are taken as $\pi / 2$. Thus we obtained $\left(\theta_{\mathcal{S}}+\theta_{\mathcal{O}} \rightarrow \pi\right)$ that is the total sum of the respective angles consistent to the light source $\mathcal{S}$ and the observer $\mathcal{O}$. If the $\mathcal{B}_{R}$ is non singular (that the domain is regular) then the Euler characteristic is $\mathcal{X}\left(\mathcal{B}_{R}\right)=1$. Hence the GBT can be rewritten as

$$
\iint_{\mathcal{B}_{R}} \mathcal{K} d S+\oint_{\partial \mathcal{B}_{R}} k d t+\epsilon_{j}=2 \pi \mathcal{X}\left(\mathcal{B}_{R}\right),
$$


here, $\epsilon_{j}=\pi$ manifest that $\gamma_{\bar{d}}$ and the total jump angle is a geodesic as the euler characteristic number is 1 symbolized by $\mathcal{X}$. Now geodesic curvature is $k\left(C_{R}\right)=\left|\nabla_{\dot{C}_{R}} \dot{C}_{R}\right|$ is to be calculated. We can evaluate the radial part of the geodesic curvature as follow:

$$
\left(\nabla_{\dot{C}_{R}} \dot{C}_{R}\right)^{r}=\dot{C}_{R}^{\psi} \partial_{\psi} \dot{C}_{R}^{r}+\Gamma_{\psi \psi}^{r}\left(\dot{C}_{R}^{\psi}\right)^{2}
$$

For large $R, C_{R}:=r(\psi)=R=$ const. where $\mathrm{R}$ represents the distance from the coordinate origin. In the last equation $\Gamma_{\psi \psi}^{r}$ gives the connections related to the optical geometry. From the above equation that first term vanishes as topological effect is not involved and the second term $k\left(C_{R}\right)=\left|\nabla_{\dot{C}_{R}} \dot{C}_{R}\right|$ will be calculated by the use of unit speed condition.

$$
\left(\nabla_{\dot{C}_{R}^{r}} \dot{C}_{R}^{r}\right)^{r} \rightarrow \frac{1}{R}
$$

$k\left(C_{R}\right) \rightarrow R^{-1}$ because there is no topological effect. By availing the advantage of the optical metric $d t^{2}=\bar{g}_{a b} d x^{a} d x^{b}$, it can be stated that $d t=R d \psi$. Hence, we can write that

$$
k\left(C_{R}\right) d t=\frac{1}{R} R d \psi
$$

All the above outcomes after combining showed the following result

$$
\iint_{\mathcal{B}_{R}} \mathcal{K} d s+\oint_{\partial \mathcal{B}_{R}} k d t=\iint_{S_{\infty}} \mathcal{K} d S+\int_{0}^{\psi+\Theta} d \psi
$$

For getting the deflection angle in the weak deflection region at 0th order the light ray obeys a straight line approximation and is expressed as $r(t)=b / \sin \psi$. The angle of deflection of photon takes the following form [22]:

$$
\gamma=-\int_{0}^{\pi} \int_{b / \sin \psi}^{\infty} \mathcal{K} \sqrt{\operatorname{det} \bar{g}} d r d \psi
$$

$\sqrt{\operatorname{det} \bar{g}}$ can be expressed as follow

$$
\sqrt{\operatorname{det} \bar{g}}=r\left(1-\frac{3 M}{r}+\frac{3 Q^{2}}{2 r^{2}}+\frac{3 a_{2} Q^{4}}{5 r^{6}}\right) d r
$$

By manipulating the above relation and inserting the values of Gaussian curvature Eq. (9) into Eq. (13), angle of deflection $\gamma$ can be stated as:

$$
\gamma \approx \frac{4 M}{b}-\frac{3 Q^{2} \pi}{4 b^{2}}+\frac{15 Q^{4} \pi}{64 b^{4}}-\frac{8 M Q^{2}}{3 b^{3}}-\frac{7 Q^{4} a_{2} \pi}{16 b^{6}}+\frac{12 M Q^{4}}{5 b^{5}}
$$

\section{GRAPHICAL INFLUENCE OF ANGLE OF DEFLECTION $\gamma$ ON NED BLACK HOLES WITH MANY HORIZONS}

In this portion, graphical behaviour of the deflection angle $\gamma$ on NED black holes with many horizons is studied. The physical significance of these plots is also demonstrated to study the impact of various parameters on the deflection angle by giving variations to $\mathrm{BH}$ charge, impact parameter and coupling constant. 
(ii)

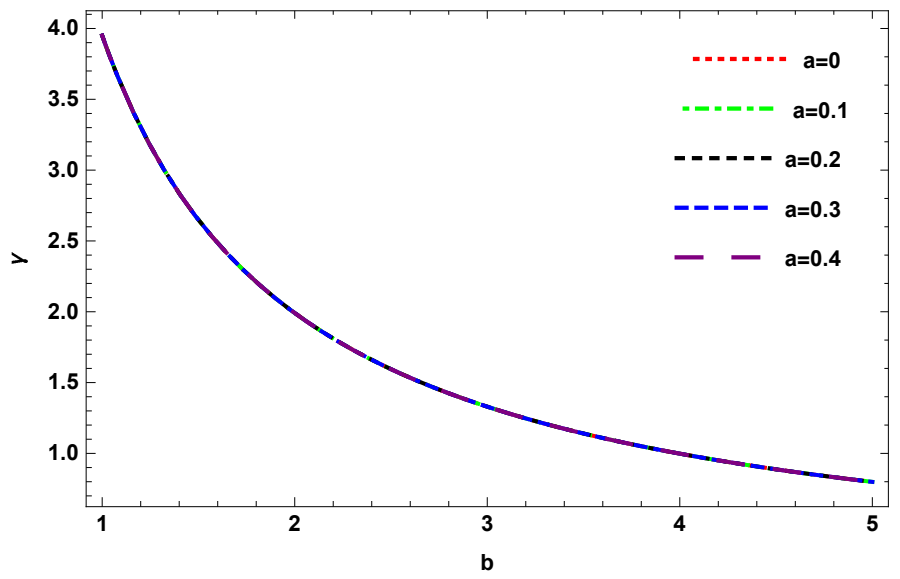

Figure 1: $\gamma$ vs $b$.

(ii) $a=$

(ii) a
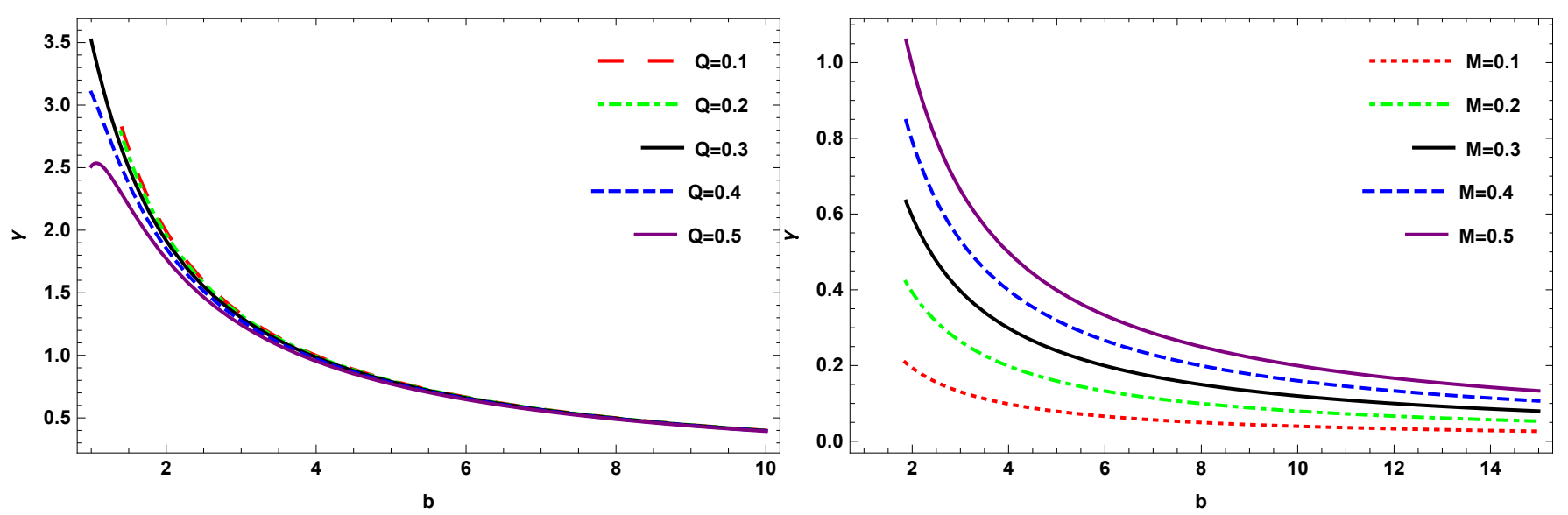

Figure 2: $\gamma$ vs $b$. 
(i

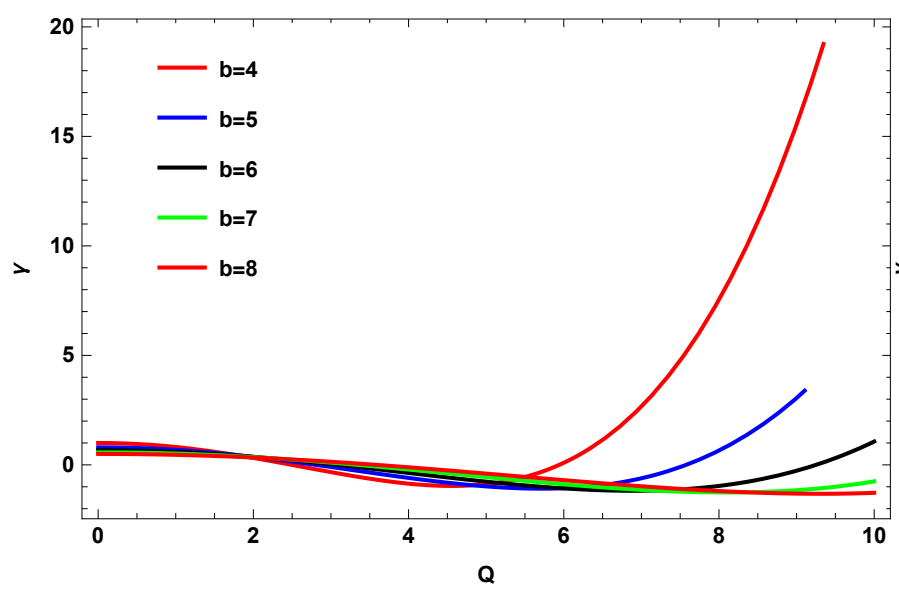

(i) $b=$

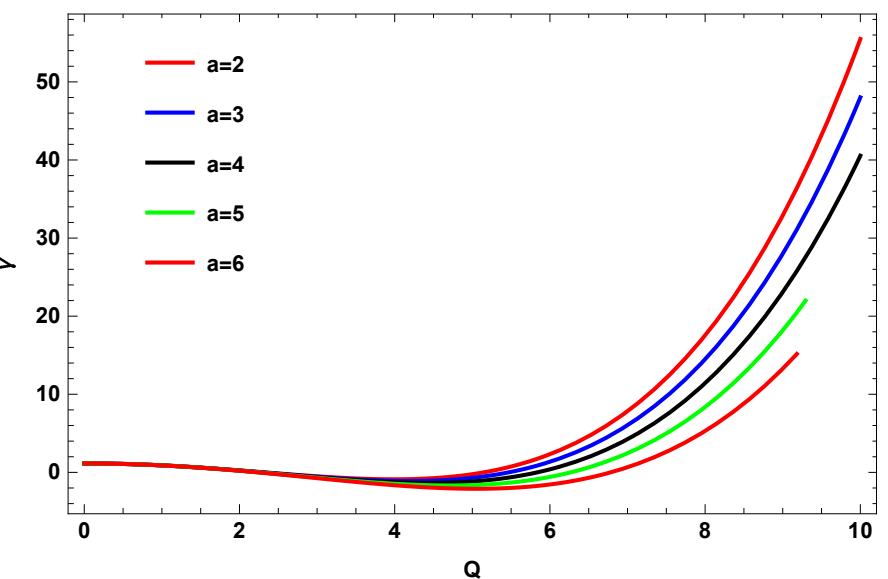

Figure 3: $\gamma$ vs $Q$.

A. Angle of Deflection $\gamma$ vs impact parameter $b$

- Fig 1 represents the behavior of $\gamma$ versus $b$ by fixing $M$ and then by fixing $Q$ and varying $a$ respectively. The domain of the impact parameter is taken to be $0 \leq b \leq 5$

1. In the left plot, it can be perceived that attained angle is constantly uniform for small variations of $a$.

2. In the right plot, it can be seen that attained angle in case of variations of $a$ is initially decreasing but as we variate the coupling constant it starts decreasing negatively when we select $a \geq 2$.

- Fig 2 presents the behavior of $\gamma$ versus $b$ by fixing $a$. It demonstrates $\gamma$ conduct for varying $Q$ and then changing the values of $M$ respectively.

1. The left graph evince that the angle of deflection in case of small variations of $Q$ is decreasing uniformly.

2. The right graph shows that angle of deflection in case of small variations of $M$ is gradually increasing .

\section{B. Deflection angle $\gamma$ vs BH charge $Q$}

- Fig 3 shows the behaviour of $\gamma$ versus $Q$ by fixing $a$ and varying $b$. It also exhibit the conduct of $\gamma$ by fixing $b$ and varying $a$ respectively.

1. In the left graph, it can be observed that angle of deflection is exponentially decreasing by giving large variation to b. $\gamma$ is rapidly declining for the variation $4<b<5$.

2. In the right graph, we examined that attained angle $\gamma$ decreases when we variate the coupling constant.

\section{EFFECT OF PLASMA ON WEAK DEFLECTION ANGLE $\gamma$ OF NED BLACK HOLES WITH MANY HORIZONS}

The section is organized basically to illustrate the impact of the angle of deflection by NED BH in plasma medium. For this desire we consider the NED BH in plasma having refractive index [103],

$$
n^{2}(r)=1-\frac{\omega_{e}^{2}(r)}{\omega_{\infty}^{2}(r)} .
$$

The corresponding refractive index is as follow

$$
n(r)=\sqrt{1-\frac{\omega_{e}^{2}}{\omega_{\infty}^{2}} Z(r)} .
$$


Here, $\omega_{e}$ represents the electron plasma frequency and $\omega_{\infty}$ represents photon frequency respectively. where the corresponding metric function is expressed as

$$
d s^{2}=-Z(r) d t^{2}+\frac{1}{Z(r)} d r^{2}+r^{2} d \Omega_{2}^{2}
$$

and

$$
Z(r)=1-\frac{2 M}{r}+\frac{Q^{2}}{r^{2}}+\frac{2 a_{2} Q^{4}}{5 r^{6}}
$$

As the source and observer both are in the tropical plane having $\left(\theta=\frac{\pi}{2}\right)$. For null geodesics we implement that $d s^{2}=0$. As before the corresponding optical metric yields that

$$
d \sigma^{2}=g_{x y}^{o p t} d x^{x} d x^{y}=n^{2}\left[\frac{d r^{2}}{Z^{2}(r)}+\frac{r^{2} d \vartheta^{2}}{Z(r)}\right]
$$

The determinant $\left(g_{x y}^{o p t}\right)$, of the above optical metric will be as follow:

$$
\sqrt{g_{x y}^{o p t}}=r\left(1-\frac{\omega_{e}^{2}}{\omega_{\infty}^{2}}\right)+M\left(3-\frac{\omega_{e}^{2}}{\omega_{\infty}^{2}}\right)-\frac{Q^{2}}{2 r}\left(3-\frac{\omega_{e}^{2}}{\omega_{\infty}^{2}}\right)-\frac{a_{2} Q^{4}}{5 r^{5}}\left(3-\frac{\omega_{e}^{2}}{\omega_{\infty}^{2}}\right) .
$$

By using Eq. (19),we can determine the following three non-zero christofell symbols

$$
\begin{gathered}
\Gamma_{00}^{0}=\left(1+\frac{\omega_{e}^{2} Z}{\omega_{\infty}^{2}}\right)\left[-Z^{\prime} Z^{-1}\left(1-\frac{\omega_{e}^{2} Z}{\omega_{\infty}^{2}}\right)-\frac{Z^{\prime} \omega_{e}^{2}}{2 \omega_{\infty}^{2}}\right] \\
\Gamma_{10}^{1}=\left(1+\frac{\omega_{e}^{2} Z}{\omega_{\infty}^{2}}\right)\left[r^{-1}\left(1-\frac{\omega_{e}^{2} Z}{\omega_{\infty}^{2}}-\frac{Z^{\prime} Z^{-1}}{2}\left(1-\frac{\omega_{e}^{2} Z}{\omega_{\infty}^{2}}\right)-\frac{Z^{\prime} \omega_{e}^{2}}{2 \omega_{\infty}^{2}}\right]\right.
\end{gathered}
$$

and

$$
\Gamma_{11}^{0}=\left(1+\frac{Z \omega_{e}^{2}}{\omega_{\infty}^{2}}\right)\left[-r Z\left(1-\frac{Z \omega_{e}^{2}}{\omega_{\infty}^{2}}\right)+\frac{r^{2} Z^{\prime}}{2}\left(1-\frac{Z \omega_{e}^{2}}{\omega_{\infty}^{2}}\right)+\frac{r^{2} Z}{2} \frac{Z^{\prime} \omega_{e}^{2}}{\omega_{\infty}^{2}}\right] .
$$

Gaussian optical curvature can be evaluated by utilizing the above Christoffel symbols and the expression for $\mathcal{K}$ can be written as follow

$$
\mathcal{K}=\frac{R_{r \psi r \psi}\left(g_{x y}^{o p t}\right)}{\operatorname{det}\left(g_{x y}^{o p t}\right)}
$$

with the help of Eq. (21) in the weak field approximation Gaussian curvature can be stated as:

$$
\begin{aligned}
\mathcal{K} & =-\frac{2 M}{r^{3}}+\frac{3 Q^{2}}{r^{4}}-\frac{6 M Q^{2}}{r^{5}}+\frac{2 Q^{4}}{r^{6}}+\frac{42 Q^{4} a_{2}}{5 r^{8}}-\frac{76 Q^{4} M a_{2}}{5 r^{9}}+\frac{\omega_{e}^{2}}{\omega_{\infty}^{2}}\left(-\frac{3 M}{r^{3}}\right. \\
& \left.+\frac{5 Q^{2}}{r^{4}}-\frac{26 M Q^{2}}{r^{5}}+\frac{10 Q^{4}}{r^{6}}-\frac{23 Q^{4} M}{r^{7}}+\frac{78 Q^{4} a_{2}}{5 r^{8}}-\frac{324 Q^{4} M a_{2}}{5 r^{9}}\right) .
\end{aligned}
$$

By utilizing GBT angle of deflection can be computed and compared with the obtained expression of angle of deflection for non-plasma. We use the fact the light beams follows a straight line approximation and compute the angle of deflection for plasma case utilize the condition at Oth order $r=\frac{b}{\sin \psi}$.

$$
\gamma=-\lim _{R \rightarrow 0} \int_{0}^{\pi} \int_{\frac{b}{\sin \psi}}^{R} \mathcal{K} d S
$$

By making use of Eq. (12) and after simplification the angle of deflection of the desired BH for plasma case can be takes the form;

$$
\begin{aligned}
\gamma & =\frac{4 M}{b}-\frac{3 \pi Q^{2}}{4 b^{2}}-\frac{8 M Q^{2}}{3 b^{3}}+\frac{15 Q^{4} \pi}{64 b^{4}}+\frac{12 M Q^{4}}{5 b^{5}}-\frac{7 Q^{4} \pi a_{2}}{16 b^{6}}+\frac{\omega_{e}^{2}}{\omega_{\infty}^{2}}\left(\frac{2 M}{b}\right. \\
& \left.-\frac{\pi Q^{2}}{2 b^{2}}+\frac{2 M Q^{2}}{b^{3}}-\frac{3 \pi Q^{4}}{16 b^{4}}-\frac{2 Q^{4} M}{3 b^{5}}-\frac{3 Q^{4} a_{2} \pi}{8 b^{6}}\right)
\end{aligned}
$$

It is obvious from the above equation that if we take $\frac{\omega_{e}^{2}}{\omega_{\infty}^{2}}=0$ then it reduces to the equation 30 and the effect of plasma is removed. 


\section{GRAPHICAL ANALYSIS FOR PLASMA MEDIUM}

The main motive of the section is to analyze the impact of plasma on the angle of deflection $\gamma$. The physical consequences of the plots are also reviewed in order to investigate the influence of plasma. Moreover, here we make a supposition i-e $\frac{\omega_{e}}{\omega_{\infty}}=10^{-1}$ and vary the impact parameter $b$, mass $M$ of the $\mathrm{BH}$, coupling constant $a_{2}$ and $\mathrm{BH}$ charge $Q$ to examine the conduct of $\gamma$ by plotting the graphs.

(ii) $Q=1$

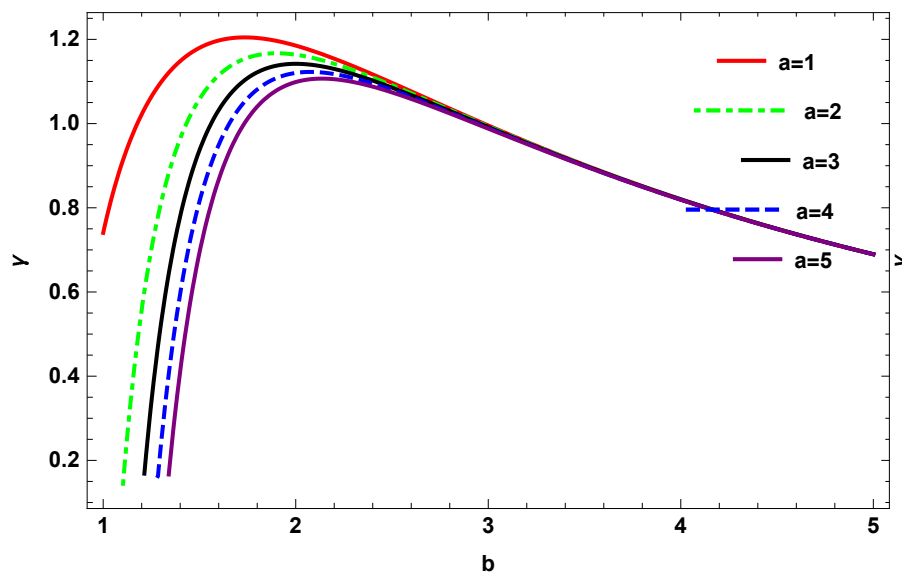

(ii) $M=$

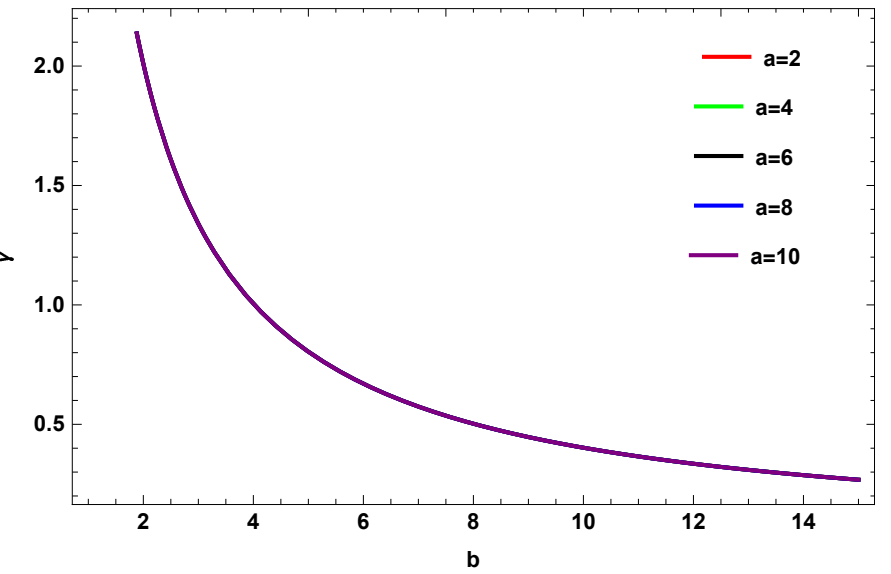

(ii) $a=5$

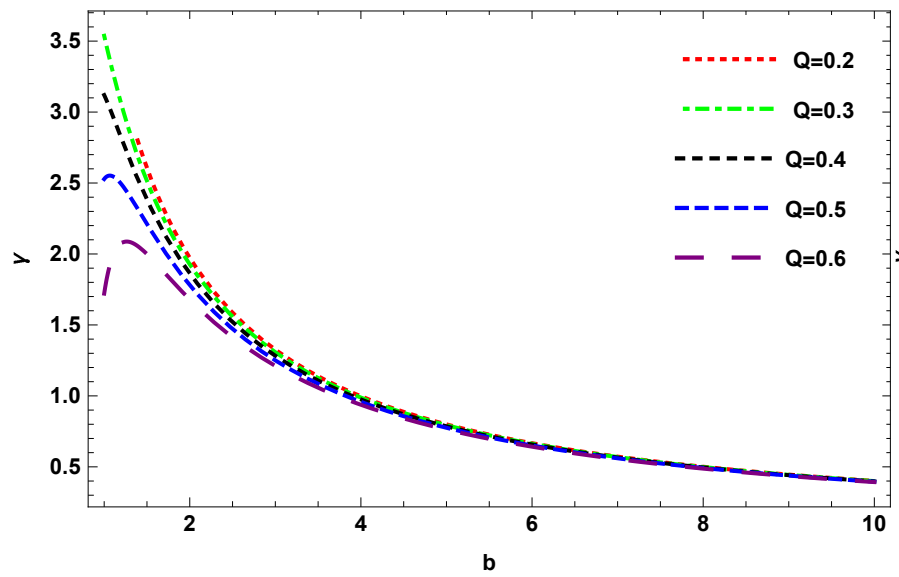

Figure 1: $\gamma$ vs $b$. (ii)

Figure $2: \gamma$ vs $b$. (ii) $b=2$

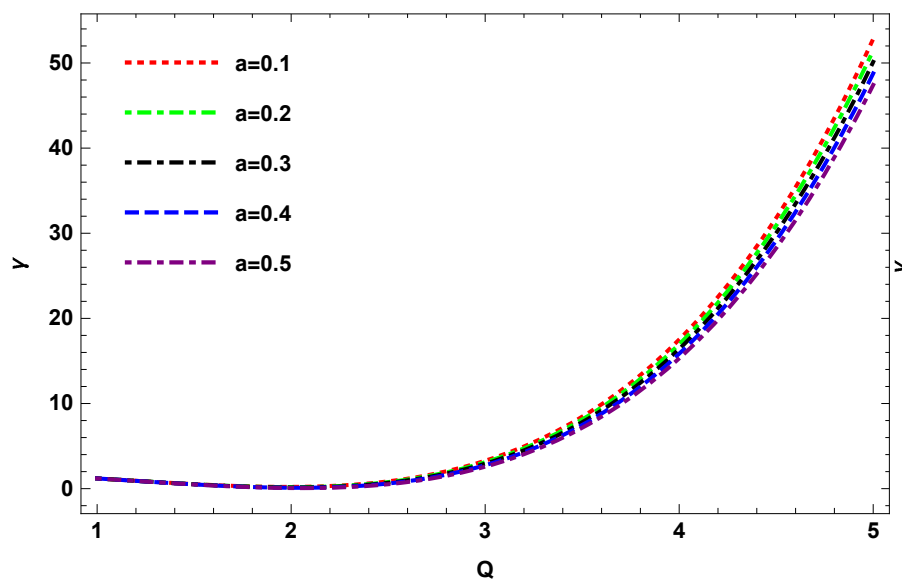

(ii) a

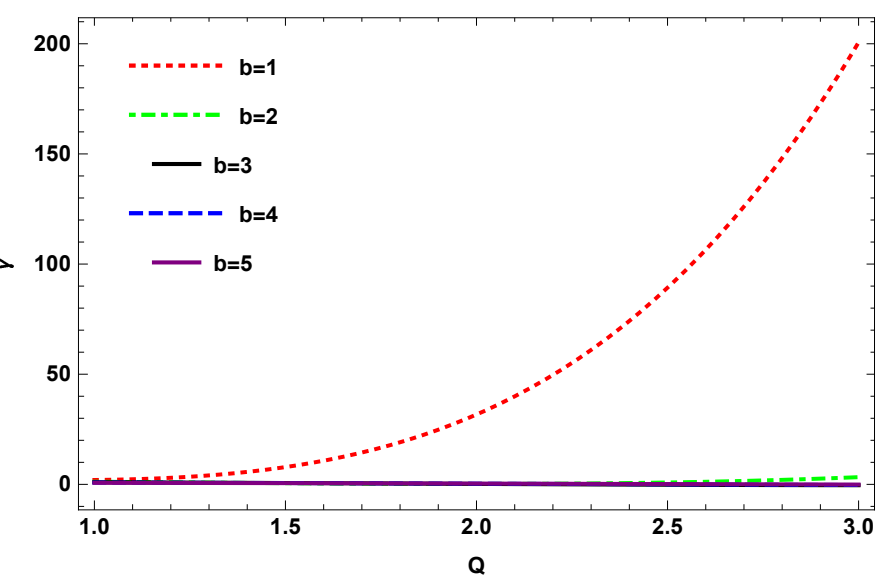

Figure 3: $\gamma$ vs $Q$. 


\section{A. Deflection angle $\gamma$ vs Impact parameter $b$}

- fig 1 exhibits the behavior of $\gamma$ versus $b$ by fixing $Q$ and $M$ and varying $a$ respectively.

1. The left graph demonstrate the phase of $\gamma$ versus $b$. The angle of deflection is declining for small values of $a$.

2. The right graph exhibits that the obtained angle $\gamma$ for fixed $M$ and small variations of $a$ is constantly uniform.

- Fig 2 shows the behavior of $\gamma$ versus $b$ by fixing $a$ and varying $Q$. It also shows the conduct of $\gamma$ by fixing $a$ and giving variation to $M$ respectively .

1. It can be examined that in the left graph the angle of deflection has small variations of $Q$ is decreasing uniformly.

2. It can be examined that in the right graph the achieved angle for small variations of $M$ with $a=5$ is gradually increasing.

\section{B. Deflection angle $\gamma$ vs BH charge $Q$}

- Fig 3 shows the behavior of $\gamma$ versus $Q$ by fixing $b$ and by varying $a$. It also shows $\gamma$ conduct by fixing $a$ and varying $b$ respectively .

1. In the left graph, it can be analyzed that angle of deflection for small variations of $a$ decreases.

2. In the right graph, it can be examined that angle of deflection is declining exponentially for small variations of $b$.

\section{COMPUTATION OF GREYBODY FACTOR OF 3}

\section{HORIZON BH}

The NED black hole with many horizons can be stated as,

$$
d s^{2}=-Z(r) d t^{2}+\frac{d r^{2}}{Z(r)}+r^{2}\left(d \theta^{2}+\sin ^{2} \theta d \phi^{2}\right),
$$

where the metric function is defined as

$$
Z(r)=1-\frac{2 M}{r}+\frac{Q^{2}}{r^{2}}+\frac{2 a_{2} Q^{4}}{5 r^{6}}
$$

The horizons of the metric $Z(r)$ are calculated numerically [21]. After the calculations, $r_{+}=1.67$ is the outer horizons of the NED black hole.

The Schrodinger like equation can be written as

$$
\left[\frac{d^{2}}{d r_{*}^{2}}+\omega^{2}-\mathrm{V}(r)\right] \psi=0
$$

and here $d r_{*}=\frac{1}{Z(r)} d r$ and $\mathrm{V}(r)$ in the above equation is expressed as

$$
V(r)=\frac{Z(r) \partial_{r} Z(r)}{r}+l^{2} \frac{Z(r)}{r^{2}}
$$

The bound of GBF is expressed as

$$
T \geq \operatorname{sech}^{2}\left(\frac{1}{2 \omega} \int_{r_{+}}^{\infty} \frac{V(r)}{Z(r)} d r_{*}\right)
$$

Here frequency is represented by $\omega$ and angular momentum is represented by $l$.

After the substitution of the potential the bound becomes

$$
T=\operatorname{sech}^{2}\left(\frac{1}{2 \omega} \int_{r_{+}}^{\infty}\left(\frac{\partial_{r} Z(r)}{r}+\frac{l(l+1)}{r^{2}}\right) d r\right)
$$


later when the integral is solved and the value of $r_{+}$is substituted we obtain,

$$
T \geq \operatorname{sech}^{2}\left[\frac{0.598802 l(l+1)+0.358564 M-0.143139 Q^{2}-0.0094645 a_{2} Q^{4}}{2 \omega}\right]
$$

Hence we have worked out the expression for the lower bound of the NED black holes with many horizons,

\section{GRAPHICAL STUDY OF THE GREY BODY BOUND OF NED BLACK HOLES WITH MANY HORIZONS}

This section is basically organized to study the graphical effect of the lower bound on NED black holes with many horizons. The physical relevance of these figures is also illustrated to analyse the effect of parameters on the rigorous bound by giving variation to the values of the coupling constant of the $\mathrm{BH}$.

\section{A. Rigorous bound $T_{b}$ vs omega $\omega$}

(i)

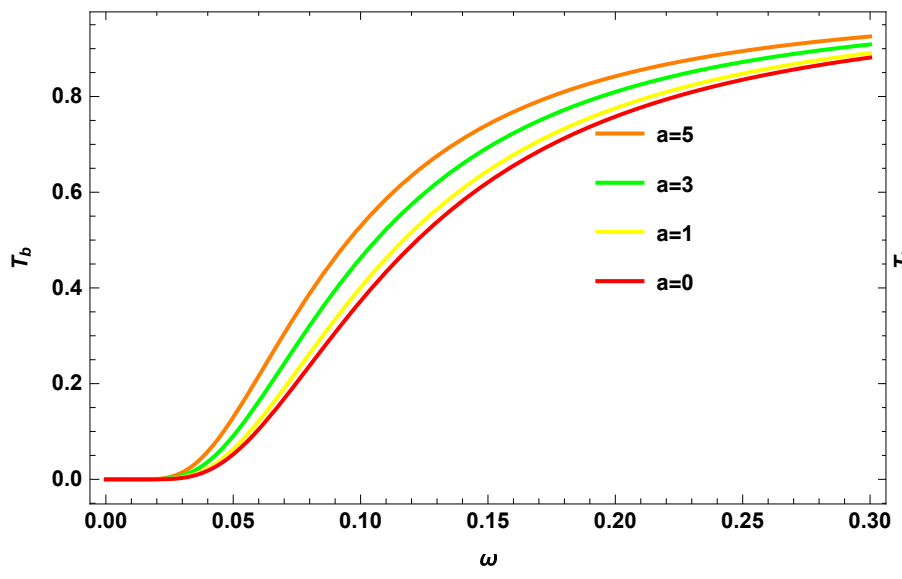

1

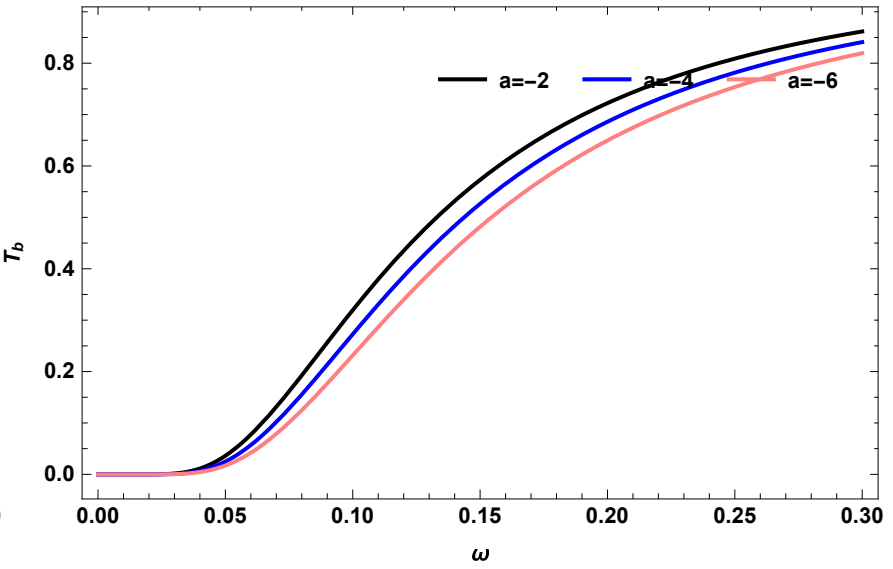

Figure 1: $T_{b}$ vs $\omega$.

- Fig 1 represents the behavior of $T_{b}$ w.r.t $\omega$ when mass $M$, charge $Q$ and $l$ are given a fixed value and changes are made in $a$ respectively. The domain of the is taken to be $0 \leq \omega \leq 0.30$

1. In the first graph, it can be observed that the bound $T_{b}$ is declining uniformly for large variations of $a$.

2. In the second graph, it can be seen that attained bound in case of negative variations of $a$ is decreasing uniformly. Hence in both the cases the grey body bound $T_{b}$ is declining.

\section{CONCLUSION}

In the present work, we immensely investigated the angle of deflection $\gamma$ for NED BH for plasma and non plasma medium. For this, we initially consider the light ray on the equatorial plane. Afterthat, we apply the theorem proposed by GW known as GBT (a new geometric technique), by using straight line approximation to obtain the angle of deflection for the desired BH. By the integration of a domain area outside the impact parameter $b$ the deflection angle $\gamma$ of light has been attained from where it depicts that GL has both the global and topological influence and is a well built technique to analyze the major singularities of $\mathrm{BH}$. We concluded that the obtained angle $\gamma$ of deflection decreases for increasing the impact parameter $b$. Hence, the computed deflection angle (30) is as follow

$$
\gamma \approx \frac{4 M}{b}-\frac{3 Q^{2} \pi}{4 b^{2}}+\frac{15 Q^{4} \pi}{64 b^{4}}-\frac{8 M Q^{2}}{3 b^{3}}-\frac{7 Q^{4} a_{2} \pi}{16 b^{6}}-\frac{16 M Q^{4}}{5 b^{5}}
$$

From the above mentioned equation we can be stated that the attained angle is based on mass, BH charge, coupling constant and on impact parameter. Also, it is clear that for the selection of $\mathrm{a}=0$ in above mentioned equation, the calculated deflection angle $\gamma$ diminish into Schwarzschild deflection angle only to the first order term. The graphical behavior of achieved angle has also 
been probed by the desired $\mathrm{BH}$. Moreover, by the consideration of homogenous plasma medium the angle of deflection of light has also been computed by desired BH for plasma case. Deflection angle of light for plasma case can be stated as:

$$
\begin{aligned}
\gamma & =\frac{4 M}{b}-\frac{3 \pi Q^{2}}{4 b^{2}}-\frac{8 M Q^{2}}{3 b^{3}}+\frac{15 Q^{4} \pi}{64 b^{4}}+\frac{12 M Q^{4}}{5 b^{5}}-\frac{7 Q^{4} \pi a_{2}}{16 b^{6}}+\frac{\omega_{e}^{2}}{\omega_{\infty}^{2}}\left(\frac{2 M}{b}\right. \\
& \left.-\frac{\pi Q^{2}}{2 b^{2}}+\frac{2 M Q^{2}}{b^{3}}-\frac{3 \pi Q^{4}}{16 b^{4}}-\frac{2 Q^{4} M}{3 b^{5}}-\frac{3 Q^{4} a_{2} \pi}{8 b^{6}}\right)
\end{aligned}
$$

in order to remove the plasma impact we neglect $\left(\frac{\omega_{e}}{\omega_{\infty}} \rightarrow 0\right)$, Eq. (31).

reduce into Eq. (30).

The graphical conduct of the obtained angle has also been demonstrated by giving variation to coupling constant, impact parameter, BH charge and to the mass. The consequences achieved from the interpretation of deflection angle stated in the paper are summed up as follows:

\section{Deflection angle $\gamma$ and Impact parameter:}

1. In our analysis the achieved angle is constantly uniform by giving small variations to $a$ and fixed $M=1$.

2. It is observed that the achieved angle for both large and small variation of coupling constant $a$ decreases gradually that exhibit the unstable behavior.

3. It is noticed that the attained angle $\gamma$ is gradually increasing by giving different variations to $\mathrm{BH}$ mass $M$.

\section{Deflection angle $\gamma$ and Black hole charge:}

1. It is examined the attained angle $\gamma$ to be exponentially declining by the small variations to impact parameter.

2. It is also noticed that attained angle $\gamma$ to be gradually declining by the small variations to coupling constant.

After that we have obtained the following expression for the bound $T_{b}$ which exhibit that the bound depends on the mass, charge, coupling constant, $\omega$ and 1 .

$$
T \geq \operatorname{sech}^{2}\left[\frac{0.598802 l(l+1)+0.358564 M-0.143139 Q^{2}-0.0094645 a_{2} Q^{4}}{2 \omega}\right]
$$

Moreover we plot the graphs of $T_{b}$ w.r.t $\omega$.

1. The first graph shows that the bound $T_{b}$ is declining uniformly for small variations of $a$.

2. The second graph manifest that attained bound in case of negative variations of $a$ is decreasing smoothly. It can be concluded that in both the situations the grey body bound $T_{b}$ is decreasing.

[1] M. Bartelmann and P. Schneider, Phys. Rep. 340, 291 (2001).

[2] V. Bozza, Phys. Rev. D 66, 103001 (2002).

[3] F. W. Dyson, A. S. Eddington, and C. Davidson, Phil. Trans. R.Soc. A 220, 291 (1920).

[4] C. M. Will, Class. Quant. Grav. 32, no.12, 124001 (2015).

[5] B. P. Abbott et al. [LIGO Scientific and Virgo], Phys. Rev. Lett. 116, no.6, 061102 (2016).

[6] M. Bartelmann, Gravitational lensing. Class. Quant. Gravit. 27, 233001 (2010).

[7] C. R. Keeton, C. S. Kochanek and E. E. Falco, Astrophys. J. 509, 561-578 (1998).

[8] E. F. Eiroa, G. E. Romero and D. F. Torres, Phys. Rev. D 66, 024010 (2002).

[9] S. Mao and B. Paczynski, Astrophys. J. 374, L37 (1991).

[10] M. Sharif and S. Iftikhar, Astrophys. Space Sci. 357, no. 1, 85 (2015).

[11] P. K. Townsend, [arXiv:gr-qc/9707012 [gr-qc]].

[12] S. M. Carroll, [arXiv:gr-qc/9712019 [gr-qc]].

[13] M. Born, Proc. Roy. Soc. Lond. A 143, no.849, 410-437 (1934).

[14] M. Born and L. Infeld, Proc. Roy. Soc. Lond. A 144, no.852, 425-451 (1934).

[15] E. S. Fradkin and A. A. Tseytlin, Phys. Lett. B 163, 123-130 (1985).

[16] K. A. Bronnikov, Phys. Rev. D 63, 044005 (2001).

[17] E. Ayon-Beato and A. Garcia, Phys. Rev. Lett. 80, 5056-5059 (1998).

[18] E. Ayon-Beato and A. Garcia, Gen. Rel. Grav. 37, 635 (2005). 
[19] S. A. Hayward, Phys. Rev. Lett. 96, 031103 (2006).

[20] S. I. Kruglov, Annals Phys. 383, 550-559 (2017).

[21] C. Gao, Phys. Rev. D 104, no.6, 064038 (2021)

[22] G.W. Gibbons and M.C. Werner, Class. Quant. Grav. 25, 235009 (2008).

[23] M.C. Werner, Gen. Relat. Gravit. 44, 3047 (2012).

[24] A. Ishihara, Y. Suzuki, T.Ono, T. Kitamura and H.Asada, Phys. Rev. D 94(8), 084015 (2016).

[25] K. Jusufi, M.C. Werner, A. Banerjee and A. Övgün, Phys. Rev. D 95(10),104012 (2017).

[26] K. de Leon and I. Vega, Phys. Rev. D 99, no.12, 124007 (2019).

[27] I. Sakalli and A. Övgün, EPL 118(6), 60006 (2017).

[28] K. Jusufi and A. Övgün, Phys. Rev. D 97(2), 024042 (2018).

[29] T. Ono, A. Ishihara and H. Asada, Phys. Rev. D 96(10), 104037 (2017).

[30] K. Jusufi, A. Övgün and A. Banerjee, Phys. Rev. D 96, no.8, 084036 (2017).

[31] A. Övgün, G. Gyulchev and K. Jusufi, Ann. Phys. 406, 152-172 (2019).

[32] K. Jusufi, I. Sakalli, and A.Ovgun, Phys. Rev.D 96 (2), 024040 (2017).

[33] H. Arakida, Gen. Relativ. Gravit.50(5), 48 (2018).

[34] T. Ono, A. Ishihara, and H. Asada, Phys. Rev. D 98(4), 044047 (2018).

[35] K. Jusufi and A. Övgün, Phys. Rev. D 97(6),064030 (2018).

[36] A. Övgün, K. Jusufi and I. Sakalli, Phys. Rev. D 99(2), 024042 (2019).

[37] K. Jusufi, A. Övgün, J. Saavedra, Y. Vasquez and P. A. Gonzalez, Phys. Rev. D 97, no.12, 124024 (2018).

[38] A. Övgün, Phys. Rev. D 98(4), 044033 (2018).

[39] A. Övgün, K. Jusufi and I. Sakalli, Ann. Phys. 399, 193 (2018).

[40] A. Övgün, Universe 5(5), 115 (2019).

[41] A. Övgün, İ. Sakallı and J. Saavedra, Annals Phys. 411, 167978 (2019).

[42] A. Övgün, I. Sakalli and J. Saavedra, JCAP 1810(10), 041 (2018).

[43] T. Ono, A. Ishihara and H. Asada, Phys. Rev. D 99(12), 124030 (2019).

[44] A. Övgün, Phys. Rev. D 99(10),104075 (2019).

[45] W. Javed, R. Babar and A. Övgün, Phys. Rev. D 99(8), 084012 (2019).

[46] A. Övgün, Phys. Lett. B 820, 136517 (2021).

[47] K. Jafarzade, M. Kord Zangeneh and F. S. N. Lobo, JCAP 04, 008 (2021).

[48] K. Takizawa, T. Ono and H. Asada, Phys. Rev. D 101, no.10, 104032 (2020).

[49] Y. Kumaran and A. Övgün, Chin. Phys. C 44, no.2, 025101 (2020).

[50] Z. Li and A. Övgün, Phys. Rev. D 101, no.2, 024040 (2020).

[51] Z. Li, G. Zhang and A. Övgün, Phys. Rev. D 101, no.12, 124058 (2020).

[52] K. Jusufi, A. Övgün, A. Banerjee and ·. I. Sakall, Eur. Phys. J. Plus 134, no.9, 428 (2019).

[53] A. Övgün, Turk. J. Phys. 44, no.5, 465-471 (2020)

[54] Y. Kumaran and A. Övgün, Turk. J. Phys. 45, 247-267 (2021)

[55] M. Okyay and A. Övgün, [arXiv:2108.07766 [gr-qc]].

[56] R. C. Pantig, P. K. Yu, E. T. Rodulfo and A. Övgün, [arXiv:2104.04304 [gr-qc]].

[57] K. Takizawa, T. Ono and H. Asada, Phys. Rev. D 102, no.6, 064060 (2020).

[58] T. Ono and H. Asada, Universe 5, no.11, 218 (2019).

[59] Q. M. Fu, L. Zhao and Y. X. Liu, Phys. Rev. D 104, no.2, 024033 (2021).

[60] A. Ishihara, Y. Suzuki, T. Ono and H. Asada, Phys. Rev. D 95, no.4, 044017 (2017).

[61] H. El Moumni, K. Masmar and A. Övgün, [arXiv:2008.06711 [gr-qc]].

[62] W. Javed, R. Babar, and A. Övgün, Phys. Rev. D 100, no.10, 104032 (2019).

[63] W. Javed, J. Abbas, and A. Övgün, Eur. Phys. J. C 79, no.8, 694 (2019).

[64] W. Javed, J. Abbas and A. Övgün, Phys. Rev. D 100, no.4, 044052 (2019).

[65] W. Javed, A. Hazma and A. Övgün,Phys. Rev. D 101, no.10, 103521 (2020).

[66] W. Javed, M. B. Khadim, A. Övgün and J. Abbas, Eur. Phys. J. Plus 135, no.3, 314 (2020).

[67] W. Javed, M. B. Khadim and A. Övgün, Eur. Phys. J. Plus 135, 595 (2020).

[68] W. Javed, A. Hamza and A. Övgün, Mod. Phys. Lett. A 35, 2050322 (2020).

[69] W. Javed, A. Hamza and A. Övgün, Universe $7,10,385$ (2021).

[70] W. Javed, J. Abbas, Y. Kumaran and A. Övgün, Int. J. Geom. Meth. Mod. Phys. 18, 2150003 (2021).

[71] W. Javed, J. Abbas and A. Övgün, Ann. Phys. 418, 168183 (2020).

[72] A. Abdujabbarov, B. Toshmatov, J. Schee, Z. Stuchlik, and B. Ahmedov, Int. J. Mod. Phys. D 26, 1741011 (2017).

[73] A. Abdujabbarov, M. Amir, B. Ahmedov, and S. G. Ghosh, Phys. Rev. D “ 93, 104004 (2016).

[74] A. Abdujabbarov, B. Ahmedov, N. Dadhich, and F. Atamurotov Phys. Rev. D “ 96, 084017 (2017).

[75] A. Abdujabbarov, B. Juraev, B. Ahmedov, and Z. Stuchlik, Astrophys. Space Sci. 361, 226 (2016).

[76] A. Abdujabbarov, B. Toshmatov, Z. Stuchlik, and B. Ahmedov, Int J. Mod. Phys. Conf. Ser. 26, 1750051 (2017).

[77] B. Turimov, B. Ahmedov, A. Abdujabbarov, and C. Bambi, Int J. Mod. Phys. D. 28, 2040013 (2019).

[78] H. Chakrabarty, A. B. Abdikamalov, A. A. Abdujabbarov, and C. Bambi, Phys. Rev. D “ 98, 024022 (2018).

[79] F. Atamurotov, B. Ahmedov, A. Abdujabbarov, Phys. Rev. D “ 92, 084005 (2015).

[80] S. Hensh, A. Abdujabbarov, J. Schee, and Z. Stuchlik, Eur. Phys. J. C “ 79, 533 (2019).

[81] S. W. Hawking, Commun. Math. Phys. 43 (1975), 199-220[erratum: Commun. Math. Phys. 46 (1976), 206].

[82] E. T. Akhmedov, V. Akhmedova and D. Singleton, Phys. Lett. B 642, 124-128 (2006). 
[83] A. Övgün and İ. Sakallı, Annals Phys. 413, 168071 (2020).

[84] W. Javed, R. Ali, R. Babar and A. Övgün, Chin. Phys. C 44, no.1, 015104 (2020).

[85] I. Sakalli and A. Övgün, Gen. Rel. Grav. 48, no.1, 1 (2016).

[86] X. M. Kuang, B. Liu and A. Övgün, Eur. Phys. J. C 78, no.10, 840 (2018).

[87] I. Sakalli and A. Ovgun, Eur. Phys. J. Plus 130, no.6, 110 (2015).

[88] W. Javed, G. Abbas and R. Ali, Eur. Phys. J. C 77, no.5, 296 (2017).

[89] S. W. Hawking, Phys. Rev. D 13 191-197 (1976).

[90] S. Fernando, Gen. Relativ. Gravit. 37, 461(2005).

[91] J. Escobedo, M.S. thesis, University of Amsterdam(2008).

[92] C. H. Fleming, (2005),http://www.physics.umd.edu/grt/taj/776b/fleming.pdf.

[93] P. Lange, M.S. thesis, Uppsala Universitet(2007).

[94] P. Boonserm and M. Visser, Annals Phys. 323, 2779-2798 (2008).

[95] P. Boonserm, [arXiv:0907.0045 [math-ph]].

[96] P. Boonserm and M. Visser, Phys. Rev. D 78, 101502 (2008).

[97] T. Ngampitipan and P. Boonserm, Int. J. Mod. Phys. D 22, 1350058 (2013).

[98] P. Boonserm, T. Ngampitipan and P. Wongjun, Eur. Phys. J. C 78, no.6, 492 (2018).

[99] P. Boonserm, T. Ngampitipan and M. Visser, JHEP 03, 113 (2014).

[100] P. Boonserm, A. Chatrabhuti, T. Ngampitipan and M. Visser, J. Math. Phys. 55, 112502 (2014).

[101] T. Ngampitipan and P. Boonserm, J. Phys. Conf. Ser. 435, 012027 (2013).

[102] P. Boonserm, T. Ngampitipan and P. Wongjun, Eur. Phys. J. C 79, no.4, 330 (2019).

[103] G. Crisnejo and E. Gallo, Phys. Rev. D 97, no.12, 124016 (2018).

[104] S. Yu and C. Gao, Int. J. Mod. Phys. D 29, no.05, 2050032 (2020). 